Supporting information for

\title{
Peptide-modified surfaces for binding carbamylated proteins from plasma
}

Yuhao Ma ${ }^{a}$, Narges Hadjesfandiari ${ }^{\mathrm{b}, \mathrm{c}}$, Michael Doschak ${ }^{\mathrm{d}}$, Dana Devine ${ }^{\mathrm{b}, \mathrm{c}}$, Marcello Tonellie, Larry Unsworth $^{\mathrm{a}, \mathrm{f}^{*}}$

a Department of Biomedical Engineering, University of Alberta, Edmonton, Canada, T6G 2R3

${ }^{\mathrm{b}}$ Department of Pathology and Laboratory Medicine, University of British Columbia, Vancouver, Canada, V6T 1 Z4

c The Centre for Blood Research, University of British Columbia, Vancouver, Canada, V6T 1Z3

d Faculty of Pharmacy and Pharmaceutical Sciences, University of Alberta, Edmonton, Canada, T6G 2R3

${ }^{\text {e }}$ Department of Medicine, University of Calgary, Calgary, Canada, T2N 1N4

${ }^{\mathrm{f}}$ Department of Chemical and Materials Engineering, University of Alberta, Edmonton, Canada, T6G 2R3

Corresponding author: Larry Unsworth (lunswort@ualberta.ca) 

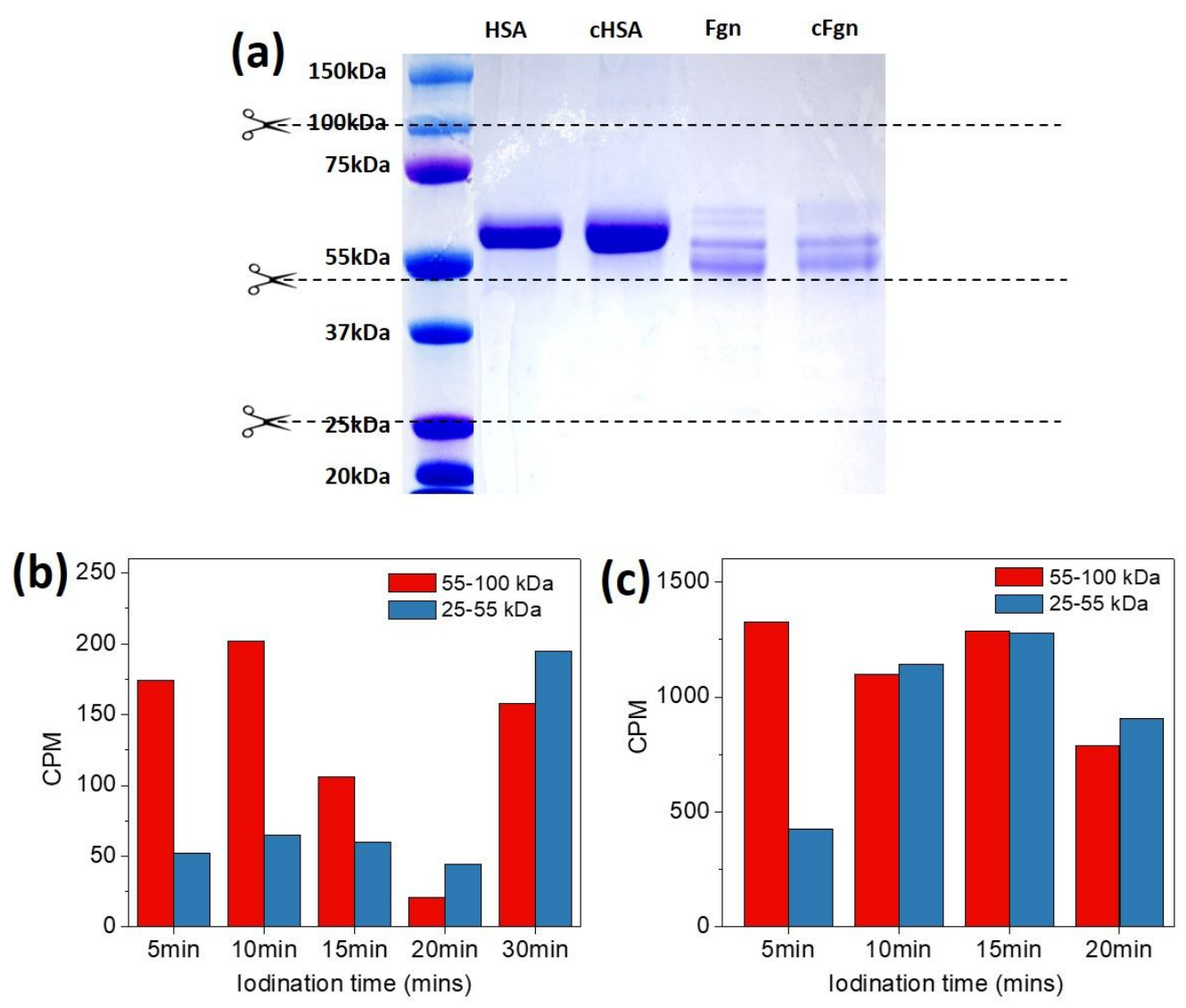

Figure S1. (a) SDS-PAGE bands of HSA/cHSA and Fgn/cFgn, (b) CPM counts for eluted fractions from 10DG column obtained by direct radiolabeling method for HSA and cHSA, (c) CPM counts for eluted fractions from 10DG column obtained by direct radiolabeling method for Fgn and cFgn. 